\title{
论 文 表面分子与超分子化学专刊
}

\section{$\operatorname{Au}(111)$ 表面一维分子组装中氢键诱导的分子轨道 能量位移}

刘婧 $^{*}$, 夏博文 ${ }^{1,2}$, 徐虎 ${ }^{2}$, 林念 ${ }^{1}$

1. 香港科技大学物理系, 香港

2. 南方科技大学物理系, 深圳 518055

*通讯作者, E-mail: jingliu@ust.hk

收稿日期：2018-06-19; 接受日期：2018-08-30; 网络版发表日期：2018-10-30

香港研究资助局(编号: N_HKUST601/15)资助项目

摘要利用扫描隧道显微术、扫描隧道谱和密度泛函理论研究了狮并[1,2-b]芴-6,12-二酮(IFDO)在Au(111)表 面形成的组装结构及其中分子轨道能级的变化. 结果表明, IFDO在Au(111)表面通过分子间氢键沿鱼骨重构结构 形成一维自组装分子链; 位于组装结构中的分子的最低未占轨道相对孤立分子向费米能级方向发生 $0.16 \sim 0.32 \mathrm{eV}$ 的位移，且位移大小与分子同周围分子形成氢键的数目和方式有关. 通过定量地对比不同氢键环境中分子的轨道 能量位移与周围分子极化能大小的变化趋势，发现周围分子的瞬时极性是造成组装结构中IFDO分子轨道能量变 化的主要因素. 而周围分子的诱导极性则对缺陷结构处分子的轨道能级有不可忽略的影响. 实验测得的IFDO分 子轨道的能量变化来自于周围分子各向异性的瞬时极性和诱导极性的共同作用.

关键词氢键, 分子轨道能量位移, 扫描隧道显微术, 扫描隧道谱, 分子极化

\section{1 引言}

如何对有机-金属界面处的分子实现精确可控的 电子结构调制是设计和构建功能性分子器件的核心问 题之一 ${ }^{[1 \sim 6]}$. 在有机-金属界面上，祄底-分子作用和分 子-分子作用均能够对有机分子的电子结构产生影 响 ${ }^{[7 ~ 13]}$. 其中, 分子间作用与分子的化学结构、吸附构 型、排列方式等多种因素有关，这为调控分子电子结 构提供了多个可调变的参数. 例如, Kröger等 ${ }^{[8]}$ 观察到 分子处于不同组装结构中时，其轨道能级发生不同程 度的位移. Willenbockel等 ${ }^{[14]}$ 研究了同种组装结构中不
同环境的分子所呈现的各异的分子轨道能级位移. Gonzalez-Lakunza等 ${ }^{[11]}$ 则发现改变分子层内分子的组 成同样可以改变分子的轨道能级. 为了探究分子间作 用调控分子轨道能级的机理, Soubatch 等 ${ }^{[12]} 、 \mathrm{Co}$ chrane 等 ${ }^{[13]}$ 先后利用微静电模型计算 ${ }^{[15]}$ 对固体表面有 机分子的轨道能级变化进行了定量研究, 揭示了周围 分子的极化能是引起被探测分子轨道能级改变的重要 因素; 这种极化能来自于实验中探测分子轨道时注入 的电荷使周围分子产生的各向异性的瞬时极性. 然而 在上述对极化能的研究中, 分子本身不因注入探测电 荷即存在的极性(包括极性分子的固有极性和因与周

引用格式: Liu J, Xia B, Xu H, Lin N. Hydrogen bond induced molecular orbital shift in one-dimensional molecular self-assemblies on Au(111). Sci Sin Chim, 2019, 49: 564-572, doi: 10.1360/N032018-00147 
围分子作用产生的诱导极性)贡献并没有被纳入考虑, 它们对于分子轨道能级的影响尚有待研究.

本文以 $\mathrm{Au}(111)$ 表面的茚并 $[1,2-b]$ 芴-6,12-二酮 (IFDO)分子体系作为模型体系, 研究了分子轨道能级 随分子间成氢键情况的变化，并且分别探讨了分子的 瞬时极性和诱导极性对分子轨道能级的影响。利用扫 描隧道显微镜(STM)观察到IFDO分子在 $\mathrm{Au}(111)$ 表面 通过分子间氢键形成一维的自组装结构. 对IFDO分子 进行扫描隧道谱(STS)测量和密度泛函理论(DFT)计 算, 发现位于组装结构中的分子的最低末占轨道 ( L U M O ) 相对孤立分子向费米能级方向有 $0.16 \sim 0.32 \mathrm{eV}$ 的位移, 且位移大小与分子同周围分子形 成氢键的数目和方式有关. 对比不同氢键环境中分子 的轨道能量位移与周围分子极化能大小的变化趋势, 发现组装结构中IFDO分子的轨道能量变化主要由周 围分子在STS探测过程中产生的瞬时极性造成. 对于 缺陷结构附近的分子，其周围分子的诱导极性同样对 其分子轨道能量产生了不可忽略的影响. 最终实验测 得的IFDO分子轨道的能量变化来自于周围分子各向 异性的瞬时极性和诱导极性的共同作用.

\section{2 实验部分}

\section{1 试剂与仪器}

IFDO (纯度99\%)购自Aldrich公司(美国)，所有实 验在超高真空STM (Omicron GmbH, 德国)上完成.

\section{2 实验方法}

实验使用的STM系统背底真空为 $10^{-10} \mathrm{mbar}$. 原子 级平整的 $\mathrm{Au}(111)$ 单晶表面通过重复循环的 $\mathrm{Ar}^{+}$轰击和 $800 \mathrm{~K}$ 退火制备. IFDO分子在 $465 \mathrm{~K}$ 下加热蒸镀到维持 在室温的干净的 $\mathrm{Au}(111)$ 表面, 即得到IFDO/Au(111)样 品. 除特殊说明外, 所有的 STM实验均在 $4.9 \mathrm{~K}$ 下进行. STM图像在恒流模式下获得, 并利用WSxM软件 ${ }^{[16]}$ 进 行处理. $\mathrm{d} I / \mathrm{d} V$ 信号通过锁相放大器获得, 所施加的正 弦调制信号频率为 $2 \mathrm{kHz}$, 振幅为 $15 \mathrm{mV}$.

采用 $\mathrm{DFT}^{[17,18]}$ 框架下的第一性原理方法，使用 $\mathrm{VASP}^{[19]}$ 软件包进行除分子极化率和偶极矩之外的计 算. 用投影缀加波(PAW)势 ${ }^{[20]}$ 来描述离子-电子作用; 用Perdew-Burke-Emzerhof (PBE)泛函 ${ }^{[21,22]}$ 框架下的广 义梯度近似 $(\mathrm{GGA})$ 来计算原子之间的电子交换关联
能. 电子波函数用平面波基矢展开, 截断能量为 $400 \mathrm{eV}$, 布里渊积分设置为 $1 \times 3 \times 1$ 的Monkhorst-Pack特殊 $\mathrm{k}$ 点 ${ }^{[23]}$. 在相邻分子层间设置 $15 \AA$ 的真空层来确保层间 去耦合. 对结构进行弛豫直至力小于 $10^{-2} \mathrm{eV} / \AA$.

IFDO分子的极化率和偶极矩由基于Gaussain09软 件 ${ }^{[24]}$ 的 $\mathrm{DFT}^{[17,18]}$ 计算得到. 所使用泛函为 $\mathrm{B} 3 \mathrm{LYP}^{[25,26]}$, 对分子内所有原子使用 6-31 $\mathrm{g}(\mathrm{d}, \mathrm{p})$ 基组. 通过内置于 Gaussain09的NBO 3.1程序对分子进行自然键轨道 $(\mathrm{NBO})^{[27,28]}$ 分析, 得到的原子电荷用于分子偶极矩的 计算.

\section{3 结果与讨论}

图1(a)显示了IFDO分子(图1(a)上部插图)蒸镀到 $\mathrm{Au}(111)$ 表面后形成的一维自组装结构. 分子沿 $\mathrm{Au}(111)$ 鱼骨重构结构排列, 在覆盖度较低时优先吸附 于fcc区域(图1(a)下部插图). 这种选择性吸附可能是由 $\mathrm{Au}(111)$ 表面鱼骨重构中 hcp 和fcc区域的电子结构差 异 ${ }^{[29]}$ 导致的. 高分辨STM图像(图1(b))显示自组装结构 中, 分子长轴平行排列, 沿分子短轴方向形成一维组装 结构. 相邻分子间通过 $\mathrm{O}$ 原子和 $\mathrm{H}$ 原子形成 4 个氢键(图 1(b)中蓝色虚线). 相邻分子间距(图1(b)白色箭头)为 $0.75 \pm 0.05 \mathrm{~nm}$, 对应氢键长度约为 $2.2 \AA$. 利用DFT方法 优化得到的真空中的一维分子组装结构模型(图1(c)) 中相邻分子间距为 $0.73 \mathrm{~nm}$, 分子间氢键长度约为 $2.3 \AA$, 与实验测量结果吻合. 同时, DFT计算得到每两 个相邻分子间的 4 个氢键能够使体系能量降低 $0.256 \mathrm{eV}$. 因此, IFDO在 $\mathrm{Au}(111)$ 表面形成的一维自组 装是由分子间氢键稳定的结构.

对 $\mathrm{Au}(111)$ 表面IFDO分子的STS测量显示了处于 不同环境的IFDO分子具有明显不同的分子轨道能量. 未处于组装结构中的孤立分子(由图2-i中黑点标示)在 约 $1.65 \mathrm{~V}$ 处出现代表分子LUMO的谱峰(图2黑色谱 线). 位于一维组装分子链链端的分子 (由图2-ii中蓝点 标示)在约 $1.49 \mathrm{~V}$ 处有LUMO峰(图2蓝色谱线). 对于一 维组装分子链内的IFDO分子 (由图2-ii中红点标示), 其 LUMO峰则出现在约 $1.33 \mathrm{~V}$ 处(图2红色谱线). 可见, 随 着与被考察分子形成氢键的分子数目增加，被考察分 子的LUMO峰位也依次向费米能级移动：以孤立分子 (与其成氢键的分子数为 0 ) 的LUMO峰位作为参考, 组 装链端部分子(与 1 个分子成氢键) 和组装链内分子 (与 2 

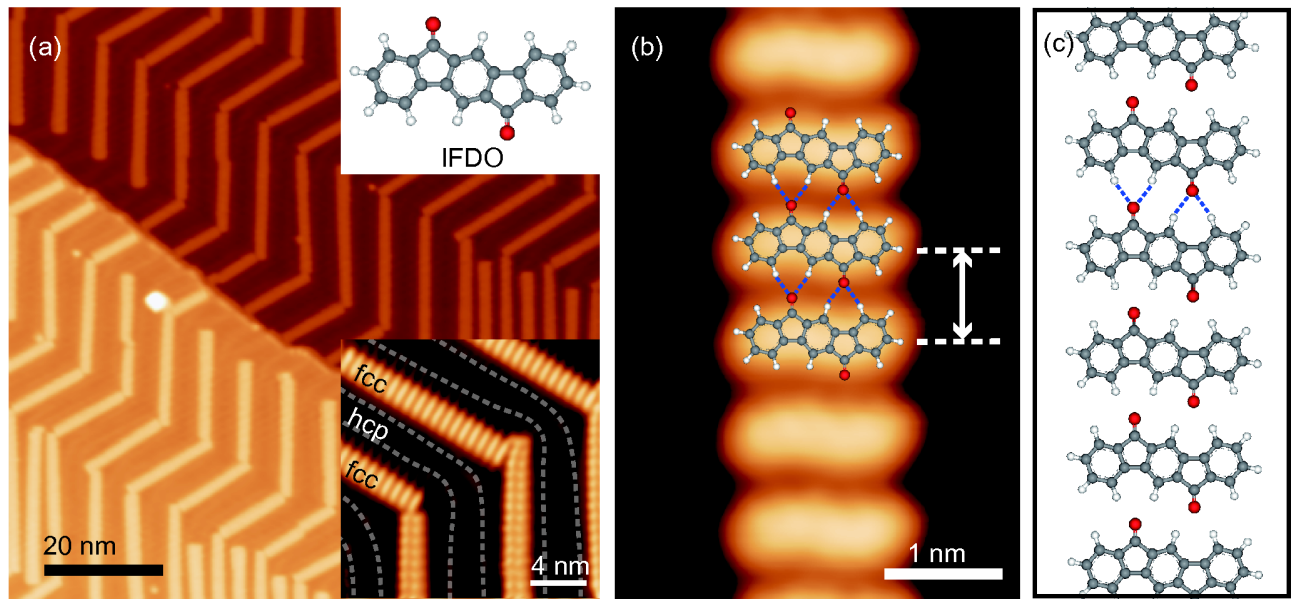

图 1 (a) IFDO分子在Au(111)表面自组装结构的STM图像. 插图: IFDO分子模型(上)和IFDO分子沿Au(111)鱼骨重构结构排 列的STM图像(下); (b) IFDO分子一维组装结构的高分辨STM图像; (c) DFT优化的真空中的IFDO一维组装结构模型. 成像条 件: (a) $-1.0 \mathrm{~V}, 0.50 \mathrm{nA}$; 插图(下): $-1.0 \mathrm{~V}, 0.30 \mathrm{nA}, 77 \mathrm{~K}$; (b) $-0.6 \mathrm{~V}, 0.30 \mathrm{nA}$ (网络版彩图)

Figure 1 (a) STM image of one-dimensional (1D) assembly of IFDO molecules on $\mathrm{Au}(111)$. Inset: A molecular model of IFDO (top), and STM image showing preferred adsorption of IFDO on fcc areas of $\mathrm{Au}(111)$ herringbone structure (bottom). (b) High-resolution STM image of the 1D assembly of IFDO. (c) DFT-optimized models of the $1 \mathrm{D}$ assembly in vacuum. Imaging condition: (a) $-1.0 \mathrm{~V}, 0.50 \mathrm{nA}$; inset (bottom) $-1.0 \mathrm{~V}, 0.30 \mathrm{nA}$, $77 \mathrm{~K}$; (b) $-0.6 \mathrm{~V}, 0.30 \mathrm{nA}$ (color online).
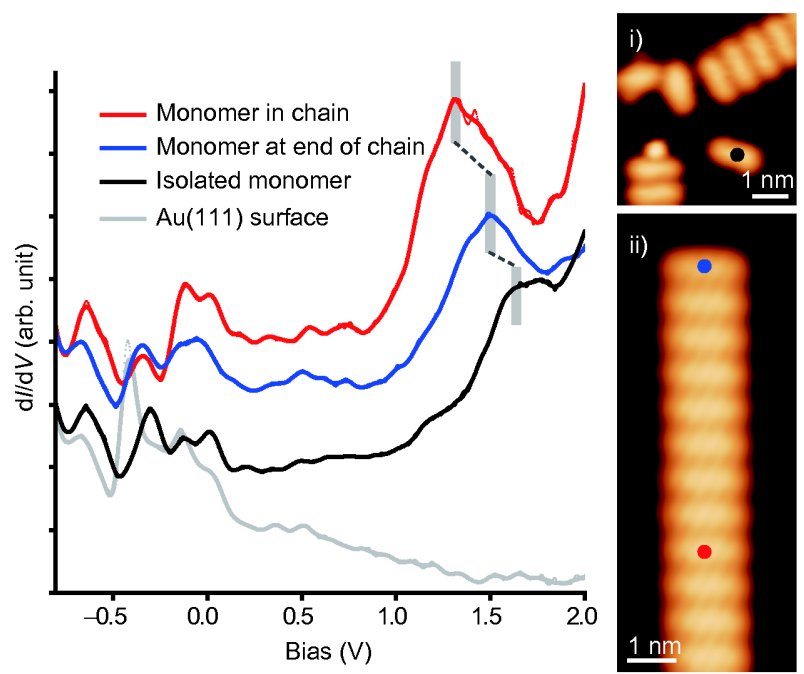

图 $2 \mathrm{Au}(111)$ 表面孤立IFDO分子(黑色), 自组装链链端分 子(蓝色), 自组装链链内分子(红色)以及空白 $\mathrm{Au}(111)$ 表面(灰 色)的 $\mathrm{d} I / \mathrm{d} V$ 谱线. 原始数据点由散点表示, Savitzky-Golay平 滑后的谱线(窗口宽度50点)由曲线表示. 插图: (i) 孤立IFDO 分子的STM图像; (ii) 自组装分子直链的STM图像. 成像条 件: (i) $-0.5 \mathrm{~V}, 0.30 \mathrm{nA}$; (ii) $1.4 \mathrm{~V}, 0.30 \mathrm{nA}$ (网络版彩图)

Figure $2 \mathrm{~d} I / \mathrm{d} V$ curves of an isolated IFDO molecule (black), a molecule at the end of a assembled molecular chain (blue), a molecule in the chain (red), and bare $\mathrm{Au}(111)$ surface (grey). Scatter plots are original data, and the curves are smoothed data by Savitzky-Golay filter (50 points in window). Inset: STM images of (i) an isolated IFDO molecule, and (ii) an assembled molecular chain on $\mathrm{Au}(111)$. Imaging condition: (i) $-0.5 \mathrm{~V}, 0.30 \mathrm{nA}$; (ii) $1.4 \mathrm{~V}, 0.30 \mathrm{nA}$ (color online).
个分子成对称的氢键) 的LUMO峰分别向费米能级移 动约 0.16 和 $0.32 \mathrm{~V}$. 同时, 对于真空中IFDO形成的一维 组装结构(图1(c))中的分子上的投影态密度(PDOS)的 DFT计算(图S1，网络版补充材料)也显示，这3种分子 的前线轨道能隙, 即LUMO和最高占据轨道(HOMO) 的能量差, 也依次变小: 孤立分子的能隙为 $1.830 \mathrm{eV}$, 链端分子能隙为 $1.756 \mathrm{eV}$, 而链内分子能隙为 $1.714 \mathrm{eV}$.

进一步地, 对一条长度为 28 个分子的自组装链(图 3(a)) 中的若干分子进行了STS测量, 以研究分子 LUMO能级位移随分子在链中位置的变化规律. 测得 的 $\mathrm{d} I / \mathrm{d} V$ 谱线显示, 相对于孤立分子的LUMO峰位置 (1.65 V, 由图3(a)中灰色虚线标示), 除了链端分子 $\left(S_{\mathrm{E}}\right)$ 出现了约 $0.18 \mathrm{~V}$ 的位移外, 第二至第六个分子 $\left(S_{2}\right.$ 、 $\left.S_{3} 、 S_{4} 、 S_{5} 、 S_{6}\right)$ 以及位于链中心的第十四个分子 $\left(S_{\mathrm{C}}\right)$ 的LUMO峰均发生了 $0.3 \mathrm{~V}$ 左右的位移. 由此说明, 自 组装链中分子的LUMO能级位移只与其成氢键的分子 数有关, 而与在其链中的具体位置无明显相关.

上述实验和理论计算结果都显示, 在IFDO/ $\mathrm{Au}(111)$ 体系中分子间氢键的形成对分子轨道能级有 明显的调制作用. 为了探究氢键诱导LUMO能级移动 的原因, 我们首先考虑了在STS测量过程中, STM针尖 向被考察分子注入电荷诱导周围分子产生的瞬时极性 对被考察分子产生的影响. 在利用STS探测分子轨道 

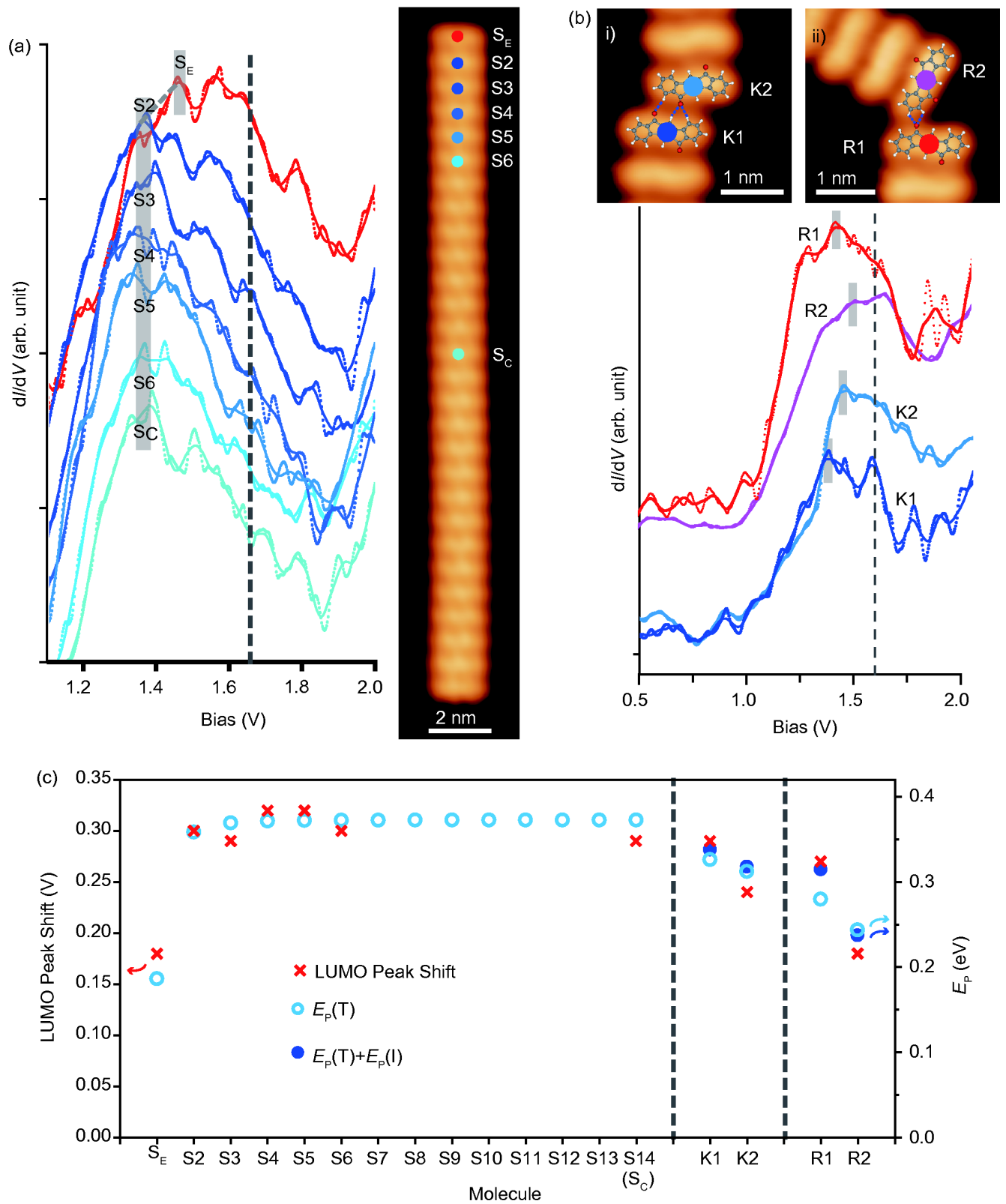

图 3 (a) 自组装分子链内不同位置分子的 $\mathrm{d} I / \mathrm{d} V$ 谱线. 插图: 自组装分子直链的STM图像. (b) 两种缺陷结构处分子的 $\mathrm{d} I / \mathrm{d} V$ 谱 线. 插图: (i) 具有平行位错的分子链的STM图像; (ii) 两条不同延伸方向的分子链交点处的STM图像. $\mathrm{d} I / \mathrm{d} V$ 原始数据点由散点 表示, Savitzky-Golay平滑后的谱线(窗口宽度50点)由曲线表示. (c) 实验测得的不同结构中分子的LUMO能级位移(红色叉号), 计算得到的 $E_{\mathrm{P}}(\mathrm{T})$ (蓝色空心圆圈), 和 $E_{\mathrm{P}}(\mathrm{T})+E_{\mathrm{P}}(\mathrm{I})$ (蓝色实心圆点). 成像条件: (a) $1.4 \mathrm{~V}, 0.30 \mathrm{nA} ;(\mathrm{b}-\mathrm{i})-0.6 \mathrm{~V}, 0.30 \mathrm{nA}$; (b-ii) $-0.6 \mathrm{~V}, 0.30 \mathrm{nA}$ (网络版彩图)

Figure 3 (a) $\mathrm{d} I / \mathrm{d} V$ curves of molecules in a straight molecular chain. Inset: STM image of a straight molecular chain. (b) $\mathrm{d} I / \mathrm{d} V$ curves of molecules in defect structures. Inset: STM images of (i) a kinked molecular chain, and (ii) a rotated molecular chain. In $\mathrm{d} I / \mathrm{d} V$ spectra, scatter plots are original data, and the curves are smoothed data by Savitzky-Golay filter (50 points in window). (c) The measured molecular LUMO shift (red crosses), and the calculated results of $E_{\mathrm{P}}(\mathrm{T})$ (blue circles)and $E_{\mathrm{P}}(\mathrm{T})+E_{\mathrm{P}}(\mathrm{I})$ (blue dots). Imaging condition: (a) $1.4 \mathrm{~V}, 0.30 \mathrm{nA}$; (b-i) $-0.6 \mathrm{~V}, 0.30 \mathrm{nA}$; (b-ii) $-0.6 \mathrm{~V}$, $0.30 \mathrm{nA}$ (color online).

时，针尖向分子注入的电荷产生的电场将使周围分子 极化，产生的瞬时极性使得被探测分子的HOMO和
LUMO都向费米能级靠近，从而减小分子前线轨道的 能隙. 利用微静电模型计算 ${ }^{[12,13,15]}$ 方法, 先分别考虑被 
考察分子周围的每个分子在注入电荷电场中被极化所 形成的各向异性的瞬时极性对被考察分子产生的作 用, 再进行加和, 就能计算周围分子瞬时极性带来的总 极化能 $E_{\mathrm{P}}(\mathrm{T})^{[13]}$ :

$$
E_{\mathrm{P}}(\mathrm{T})=\frac{\mathrm{e}^{2}}{16 \pi^{2} \epsilon_{0}^{2}} \sum_{i=1}^{N-1}\left(\alpha_{\mathrm{L}} \cos ^{2} \beta_{i}+\alpha_{\mathrm{S}} \sin ^{2} \beta_{i}\right) \frac{1}{R_{i}{ }^{4}}
$$

其中, $\alpha_{\mathrm{L}}=60.280 \AA^{3}$ 和 $\alpha_{\mathrm{S}}=33.718 \AA^{3}$ 分别为IFDO分子长 轴和短轴的极化率; $R_{i}$ 为被考察分子中心和其周围第 $i$ 个分子中心的间距, $\beta_{i}$ 则为二者中心连线与第 $i$ 个分子 长轴间的夹角. $R_{i}$ 和 $\beta_{i}$ 均通过对 $\mathrm{STM}$ 图像进行测量来 确定.

利用式(1)计算得到的长度为 28 个分子的分子链 中第一个分子 $\left(S_{\mathrm{E}}\right)$ 到第 14 个分子 $\left(S_{\mathrm{C}}\right)$ 的 $E_{\mathrm{P}}(\mathrm{T})$ 由图 $3(\mathrm{c})$ 中 的蓝色圆圈表示. 可见, 除了链端分子 $\left(S_{\mathrm{E}}\right)$ 感受到的瞬 时极性极化能较小 $(0.1864 \mathrm{eV})$ 外，从第二个分子 $\left(S_{2}\right)$ 到 链中心分子 $\left(S_{\mathrm{C}}\right)$ 均感受到 $0.37 \mathrm{eV}$ 左右的极化能. 这一 变化规律和实验测得的分子链中分子LUMO能级相对 孤立分子的位移随其在链中位置的变化趋势(图3(c)中 红色叉号)一致, 说明瞬时极性极化能模型能较好地解 释一维组装链中链端分子和链内分子相对孤立分子 LUMO能级的位移.

除了直分子链外, IFDO分子在 $\mathrm{Au}(111)$ 表面的组 装还存在一些缺陷结构，其中较为常见的两类为图3 (b)-i中显示的分子链中的平行位错, 以及图3(b)-ii中显 示的不同延伸方向分子链的交汇处. 缺陷结构处的分 子虽然也可以与旁边分子形成氢键(图3(b)-i和 ii 中蓝 色虚线标示), 但其与两侧分子形成氢键的方式及数目 均与直链中的分子不同. 我们测量了这两种缺陷结构 (图3(b)-i和ii)处的分子(K1、K2、R1、R2)的LUMO能 级位移(由图3(c)中相应红叉标示), 并利用式(1)计算了 它们所感受到的来自周围分子的瞬时极性极化能(计 算中考虑缺陷两侧的分子链各含 10 个分子), 结果用图 $3(\mathrm{c})$ 中相应蓝色圆圈标示. 与直链中分子的情况不同 的是, 两种缺陷结构中分子感受到的瞬时极性极化能 变化趋势并不能与实验测得的LUMO能级位移很好地 吻合. 一方面，计算得到 $\mathrm{K} 1$ 和 $\mathrm{K} 2$ 具有相近的 $E_{\mathrm{P}}(\mathrm{T})$ 值, 但实验测得 K1 的LUMO峰位移(约 $0.29 \mathrm{~V}$ ) 明显大于 K2 (约 $0.24 \mathrm{~V}$ ). 另一方面, 计算得到的 $\mathrm{R} 1$ 和 $\mathrm{R} 2$ 的 $E_{\mathrm{P}}(\mathrm{T})$ 值差 异也不足以解释STS实验测得的二者较大的LUMO峰 位移差(约 $0.09 \mathrm{~V}$ ).
为了理解上述 $E_{\mathrm{P}}(\mathrm{T})$ 值变化趋势和实验结果的差 异, 我们在瞬时极性极化能的基础上, 又考虑了周围分 子被氢键诱导而出现的诱导极性对被考察分子轨道能 级的影响. 对于吸附在 $\mathrm{Au}(111)$ 表面孤立的IFDO分子, 其 $C_{2 h}$ 对称性使得分子在其骨架平面内长轴和短轴方 向都不存在固有偶极. 而当一个IFDO分子与其他分子 形成氢键时, 氢键作用将使得分子内的电荷重新排布. 为了考察这种氢键导致的分子内电荷分布的变化, 我 们以 5 分子体系为例, 计算了其真空中形成氢键和未 形成氢键情况下的电荷密度变化 $\mathrm{d} \rho$ :

$$
\mathrm{d} \rho=\rho_{\text {pentamer }}-5 \rho_{\text {monomer }}
$$

其中, $\rho_{\text {pentamer }}$ 为通过氢键组装形成的 5 分子结构中分子

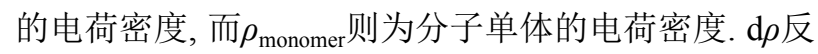
映了由氢键形成而导致的分子内电荷密度重新分布的 情况. 计算得到的 $\mathrm{d} \rho$ 的空间分布显示在图4(a)中, 其中 蓝色和红色分别表示因形成氢键而引起电荷密度减小 和增加的区域. 由图4(a)可见, 氢键的形成使分子内的 电荷发生了明显的重新分布. 对于一维组装链内的分 子, 由于其与两侧分子形成对称的氢键，因此其电荷 密度重新分布的情况也呈现 $C_{2 h}$ 对称性. 这导致形成氢 键后, 组装链内分子在分子平面内任意方向都不体现 诱导偶极矩. 但对于位于链端的分子, 分子两侧不对 称的氢键使得分子具有了一定的诱导偶极矩. 对一维 排列的3分子体系(图4(b))中分子偶极矩的计算验证了 上述结论. 在图 4(b) 所示的3分子结构中, 中央分子 $\left(S_{\mathrm{C}}\right)$ 长轴及短轴的诱导偶极矩都为 0 , 但端部分子 $\left(S_{\mathrm{E}}\right)$ 则在 长轴短轴方向都具有一定的诱导偶极矩(图4(b)中蓝色 数字和箭头标注). 其中, 链端分子上对于链内其他分 子起主要作用的短轴偶极矩大小为 $0.0043 e \AA$, 相比其 在STS实验中产生的瞬时极性在同一方向上的等效偶 极矩大小 $(0.5695 e \AA)$ 而言可以忽略. 因此直链链端分 子的诱导极性作用几乎可以忽略，这使得仅用瞬时极 性极化能 $E_{\mathrm{P}}(\mathrm{T})$ 就能较好地解释直链链端和链内分子 的LUMO能级位移.

对于其他与周围分子形成不对称氢键的分子, 如 图3(b)-i和ii所示的缺陷结构处的分子K1、K2、R1和 $\mathrm{R} 2$, 由氢键诱导产生的诱导偶极矩则可能对其附近分 子的能级产生不可忽略的影响. 图4(c,d)展示了利用 简化的模型计算得到的真空中这两类缺陷结构处分子 的诱导偶极矩的方向及大小. 利用诱导偶极矩计算结 

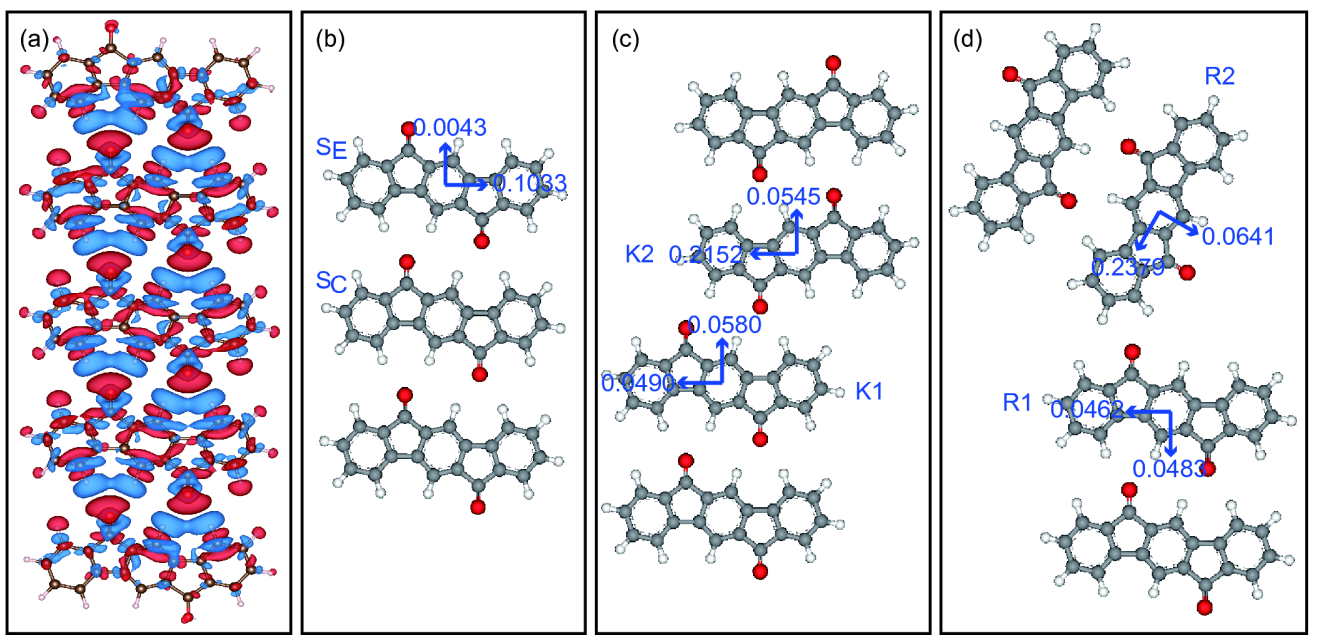

图 4 (a) 5 分子形成的一维氢键组装结构的电荷得失密度图, 等密度面处电荷密度为 $2.53769 \times 10^{-4} e$. (b) 3 分子的直链结构模 型. 蓝色箭头和数字标示了链端分子诱导偶极矩的大小和方向. (c) 4 分子的平行位错结构模型. 蓝色箭头和数字标示了 $\mathrm{K} 1$ 、 $\mathrm{K} 2$ 分子的诱导偶极矩的大小和方向, 单位为 $e \AA$. (d) 4 分子的不同延伸方向分子链交点处结构模型. 蓝色箭头和数字标示了 R1、R2分子的诱导偶极矩的大小和方向(网络版彩图)

Figure 4 (a) Differential charge density of a straightly assembled pentamer (iso-surface density: $2.53769 \times 10^{-4} e$ ). Simplified models of (b) a straight molecular chain formed by 3 molecules, (c) a kinked chain formed by 4 molecules, and (d) a rotated chain formed by 4 molecules with induced dipole moments (in $e \AA$ ) of molecules marked (color online).

果，能够根据式(3)计算分子的诱导极性使其附近被探 测分子感受到的诱导极性极化能 $E_{\mathrm{P}}(\mathrm{I})$ :

$$
E_{\mathrm{P}}(\mathrm{I})=\frac{e}{4 \pi \epsilon_{0} r^{2}}\left(P_{\mathrm{L}} \cos \gamma_{\mathrm{L}}+P_{\mathrm{S}} \cos \gamma_{\mathrm{S}}\right)
$$

其中, $P_{\mathrm{L}}$ 和 $P_{\mathrm{S}}$ 分别为分子长轴和短轴方向上的诱导偶 极矩大小; $r$ 为两分子中心间距; $\gamma_{\mathrm{L}}$ 和 $\gamma_{\mathrm{S}}$ 分别为两分子 连线方向(由具有诱导极性的分子指向被考察分子)与 分子长轴和短轴偶极矩方向夹角. $r 、 \gamma_{\mathrm{L}}$ 和 $\gamma_{\mathrm{S}}$ 均通过对 STM图像进行测量来确定(图S2). 由此计算出的诱导 极性极化能 $E_{\mathrm{P}}(\mathrm{I})$ 和瞬时极性极化能 $E_{\mathrm{P}}(\mathrm{T})$ 共同作用于 被考察分子, 使被考察分子的LUMO能级发生变化. 以 不同延伸方向分子链交汇处(图3(b)-ii)的R1分子为例， 其感受到周围分子的瞬时极性极化能为 $E_{\mathrm{P}}(\mathrm{T})=$ $0.2799 \mathrm{eV}$ (图3(c)中R1对应的蓝色空心圆圈), 而R2分 子被氢键诱导形成的诱导极性则额外贡献了 $E_{\mathrm{P}}(\mathrm{I})=$ $0.0348 \mathrm{eV}$ 的极化能, 因而 $\mathrm{R} 1$ 分子感受到的总极化能为 $E_{\mathrm{P}}(\mathrm{T})+E_{\mathrm{P}}(\mathrm{I})=0.3147 \mathrm{eV}$ (图3(c)中 $\mathrm{R} 1$ 对应的蓝色实心圆 点). 图3(c)中以蓝色实心圆点标示了缺陷结构附近的4 种分子 $\mathrm{K} 1 、 \mathrm{~K} 2 、 \mathrm{R} 1$ 和 $\mathrm{R} 2$ 受到的加入 $E_{\mathrm{P}}(\mathrm{I})$ 修正后的总 极化能, 即 $E_{\mathrm{P}}(\mathrm{T})+E_{\mathrm{P}}(\mathrm{I})$. 比较 $E_{\mathrm{P}}(\mathrm{T})$ (图3(c)中蓝色圆圈), $E_{\mathrm{P}}(\mathrm{T})+E_{\mathrm{P}}(\mathrm{I})$ (图3(c)中蓝色实心圆点) 与 $\mathrm{STS}$ 实验测得 的LUMO能级位移(图3(c)中红叉)的变化趋势可以看
出, 经过 $E_{\mathrm{P}}(\mathrm{I})$ 修正后的总极化能能够更好地描述分子 LUMO能级的变化. 由此可知, 被考察分子周围分子 的瞬时极性所产生的极化能 $E_{\mathrm{P}}(\mathrm{T})$ 是导致IFDO 分子 LUMO能级移动的主要原因; 但在缺陷结构处, 周围分 子的诱导极性产生的极化能 $E_{\mathrm{P}}(\mathrm{I})$ 对被考察分子能级的 移动也有不可忽略的贡献. 需要特别说明的是, 两种缺 陷结构中, 除了最临近缺陷的分子(K1、K2、R1、R2) 外, 其他分子均能够与两侧分子对称地形成氢键, 它们 的诱导偶极矩大小可以忽略, 因此在计算 $E_{\mathrm{P}}(\mathrm{I})$ 时未作 考虑.

上述极化能计算均将分子抽象为分子层平面内按 照一定规律排列并具有特定极性的点, 并且没有考虑 分子处于不同环境中时构型的差异. 同时, 极化能的 计算结果也无法反映金属祄底对分子轨道能级产生的 影响, 包括镜像势的作用、祄底-分子间电荷转移

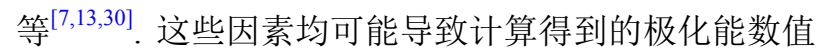
与实验测量结果间的差异. 但上述结果显示, 总极化能 $E_{\mathrm{P}}(\mathrm{T})+E_{\mathrm{P}}(\mathrm{I})$ 的变化趋势能很好地反映IFDO分子处于 不同环境中时LUMO能级的位移, 这对预测和理解实 验测得的分子轨道能量位移有充分的参考作用.

最后, 我们排除了分子间电子离域对IFDO分子轨 道能级的影响. 有机-金属界面处的有机分子通过具有 


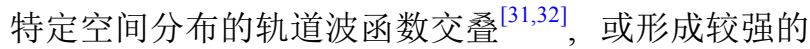
分子间氢键从而使得分子中 $\pi$ 体系内的电子能够通过 氢键区域在更大的范围内流动 ${ }^{[33,34]}$ ，二者均可能发生 分子间的电子离域, 从而导致分子电子结构的变化. 在 $\mathrm{Au}(111)$ 表面的IFDO体系中，若分子的轨道能量变 化由分子间的电子离域引起，那么通过破坏部分分子 的共轭，将能够减小电子离域范围，从而使得IFDO分 子轨道的能量位移减小. 基于此, 为了检验 $\mathrm{Au}(111)$ 表 面IFDO体系中分子间电子离域的影响, 我们利用两个 反向排列的甲酫分子来代替一个IFDO分子, 并使甲酫 分子对与IFDO分子间隔排列形成一维分子链(图5(a)), 来模拟破坏了分子链中 $1 / 2$ 分子的共轭骨架的情况. 在 这样的IFDO-甲醛分子链中，IFDO和甲醛间形成的氢 键(图5(a)中蓝色虚线标示)与纯IFDO分子链中的分子 间氢键具有类似的排列方式和长度 $(2.3 \sim 2.4 \AA)$, 但甲 醛分子对处不再存在共轭的分子结构. 对这一结构中 分子上的PDOS进行DFT计算(图5(b)), 结果显示其中 的IFDO分子的LUMO能级仍相对于孤立IFDO分子发 生明显的向费米能级方向的移动 $\left(\left|\Delta E_{\mathrm{LUMO}}\right|=0.137 \mathrm{eV}\right)$, 且HOMO-LUMO能隙为 $1.691 \mathrm{eV}$, 与位于纯IFDO分子 链内的分子能隙 $(1.714 \mathrm{eV})$ 接近, 而明显小于孤立 IFDO分子的能隙 $(1.830 \mathrm{eV})$. 由此可见, 破坏了分子链 中部分分子的共轭对分子能级移动没有明显影响。以 上结果说明, 分子间的电子离域并非是引起 $\mathrm{Au}(111)$ 表 面IFDO体系中分子轨道能级位移的主要原因.

\section{4 结论}

本文利用低温STM观察到IFDO分子在Au(111)表 面沿鱼骨重构结构形成由分子间氢键稳定的一维自 组装分子链. 对组装结构中分子的STS测量显示, 组装 结构中IFDO分子的LUMO能级相比表面吸附的孤立 分子向费米能级发生明显的移动，且能级位移的大小 依赖于IFDO分子与周围分子形成氢键的方式和数目: 在分子形成的直链中，链端分子只与一个分子形成氢 键, 其LUMO峰位移为约 $0.16 \mathrm{~V}$; 链内分子与两侧两个 分子形成对称的氢键, 其LUMO峰位移为约 $0.32 \mathrm{~V}$; 而 缺陷结构处分子与两侧两个分子形成不对称的氢键, 它们的LUMO峰位移处于 $0.16 \sim 0.32 \mathrm{~V}$. 极化能的计算

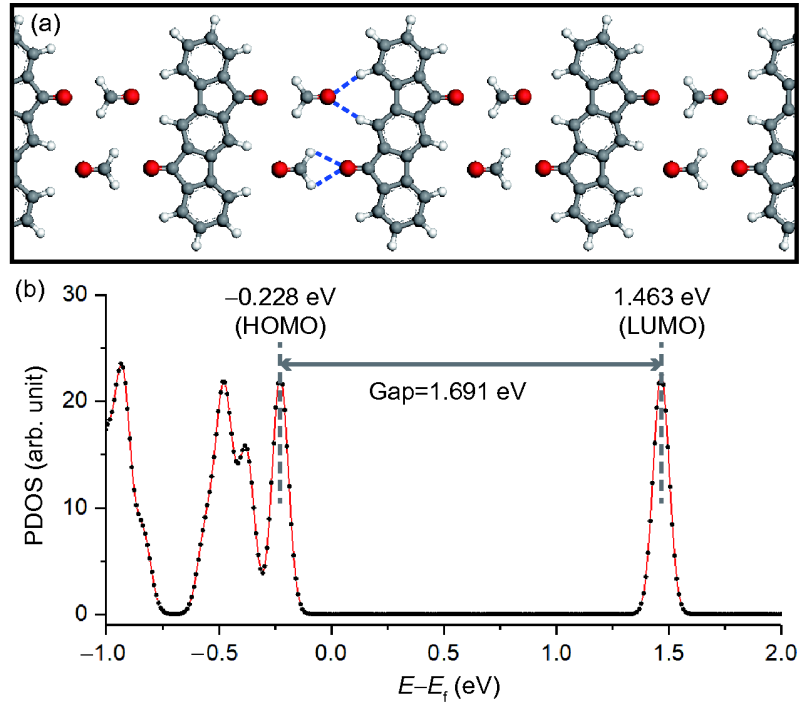

图 5 (a) 由IFDO分子与甲醛分子交替排列形成的一维组装 结构的优化模型. (b) 计算得到的IFDO-甲醛分子链中IFDO 分子上的投影态密度曲线(网络版彩图)

Figure 5 (a) Optimized models of the one-dimensional structure formed by alternately arranged IFDO molecules and formaldehydes. (b) Calculated PDOS of IFDO molecule in IFDO-formaldehyde molecular chains (color online).

结果显示, IFDO分子轨道能量移动主要由周围分子 的极化作用造成. 其中, STS测量过程中针尖注入的 电荷导致周围分子产生的瞬时极性贡献了降低 LUMO能级的大部分极化能，而由于分子处于不对称 的氢键环境中而产生的诱导极性则对缺陷结构附近 分子的轨道能量变化具有不可忽略的贡献. 在 $\mathrm{Au}(111)$ 表面的IFDO分子体系中, 分子间氢键对于分 子轨道能量变化的作用体现在两方面: (1) 分子间通 过形成氢键相互靠近, 这使对距离十分敏感的瞬时极 性能够发挥其对分子轨道能级的调制作用; (2) 在缺 陷结构附近的分子因与周围分子形成不对称的氢键 而被诱导出现各向异性的极性，由此对其周围分子的 轨道能级产生影响. 上述结果显示了利用分子间氢键 调制有机分子电子结构的可能性. 同时，本文也揭示 了除瞬时极性外，周围分子的诱导极性对分子轨道能 量同样有可观的调制作用. 通过分子间作用聚集在一 起的分子的电子结构变化可能来自于这几种不同的 分子极性的共同作用. 


\section{补充材料}

本文的补充材料见网络版http://chemen.scichina.com. 补充材料为作者提供的原始数据, 作者对其学术质量和内容负责.

\section{参考文献}

1 Dimitrakopoulos CD, Malenfant PRL. Adv Mater, 2002, 14: 99-117

2 Kahn A, Koch N, Gao W. J Polym Sci B, 2003, 41: 2529-2548

3 Kulkarni AP, Tonzola CJ, Babel A, Jenekhe SA. Chem Mater, 2004, 16: 4556-4573

4 Koch N. ChemPhysChem, 2007, 8: 1438-1455

5 Grätzel M. Acc Chem Res, 2009, 42: 1788-1798

6 Facchetti A. Chem Mater, 2011, 23: 733-758

7 Hesper R, Tjeng LH, Sawatzky GA. Europhys Lett, 1997, 40: 177-182

8 Kröger J, Jensen H, Berndt R, Rurali R, Lorente N. Chem Phys Lett, 2007, 438: 249-253

9 Kilian L, Hauschild A, Temirov R, Soubatch S, Schöll A, Bendounan A, Reinert F, Lee TL, Tautz FS, Sokolowski M, Umbach E. Phys Rev Lett, 2008, 100: 136103

10 Franke KJ, Schulze G, Henningsen N, Fernández-Torrente I, Pascual JI, Zarwell S, Rück-Braun K, Cobian M, Lorente N. Phys Rev Lett, 2008, 100: 036807

11 Gonzalez-Lakunza N, Canas-Ventura ME, Ruffieux P, Rieger R, Mullen K, Fasel R, Arnau AÃ. ChemPhysChem, 2009, 10: 2943-2946

12 Soubatch S, Weiss C, Temirov R, Tautz FS. Phys Rev Lett, 2009, 102: 177405

13 Cochrane KA, Schiffrin A, Roussy TS, Capsoni M, Burke SA. Nat Commun, 2015, 6: 8312

14 Willenbockel M, Stadtmüller B, Schönauer K, Bocquet FC, Lüftner D, Reinisch EM, Ules T, Koller G, Kumpf C, Soubatch S, Puschnig P, Ramsey MG, Tautz FS. New J Phys, 2013, 15: 033017

Silinsh EA. Organic Molecular Crystals: Their Electronic States. Berlin: Springer, 1980

Horcas I, Fernández R, Gómez-Rodríguez JM, Colchero J, Gómez-Herrero J, Baro AM. Rev Sci Instrum, 2007, 78: 013705 Caricato M, Li X, Hratchian HP, Izmaylov AF, Bloino J, Zheng G, Sonnenberg JL, Hada M, Ehara M, Toyota K, Fukuda R, Hasegawa J, Ishida M, Nakajima T, Honda Y, Kitao O, Nakai H, Vreven T, J. A. Montgomery J, Peralta JE, Ogliaro F, Bearpark M, Heyd JJ, Brothers E, Kudin KN, Staroverov VN, Kobayashi R, Normand J, Raghavachari K, Rendell A, Burant JC, Iyengar SS, Tomasi J, Cossi M, Rega N, Millam JM, Klene M, Knox JE, Cross JB, Bakken V, Adamo C, Jaramillo J, Gomperts R, Stratmann RE, Yazyev O, Austin AJ, Cammi R, Pomelli C, Ochterski JW, Martin RL, Morokuma K, Zakrzewski VG, Voth GA, Salvador P, Dannenberg JJ, Dapprich S, Daniels AD, Farkas O, Foresman JB, Ortiz JV, Cioslowski J, Fox DJ. Gaussian 09, Revision A.02. Wallingford CT: Gaussian Inc., 2009

Becke AD. J Chem Phys, 1993, 98: 5648-5652

Reed AE, Curtiss LA, Weinhold F. Chem Rev, 1988, 88: 899-926

Glendening ED, Reed AE, Carpenter JE, Weinhold F. NBO, 3.1. Madison: University of Wisconsin, Theoretical Chemistry Institute, 1996

Chen W, Madhavan V, Jamneala T, Crommie MF. Phys Rev Lett, 1998, 80: 1469-1472

Dougherty DB, Maksymovych P, Lee J, Yates Jr. JT. Phys Rev Lett, 2006, 97: 236806

Kowalzik P, Atodiresei N, Gingras M, Caciuc V, Schnaebele N, Raimundo JM, Blügel S, Waser R, Karthäuser S. Phys Chem Chem Phys, 2012 , 
14: $1635-1641$

\title{
Hydrogen bond induced molecular orbital shift in one-dimensional molecular self-assemblies on Au(111)
}

\author{
Jing Liu ${ }^{1 *}$, Bowen $\mathrm{Xia}^{1,2}, \mathrm{Hu} \mathrm{Xu}^{2}$, Nian $\mathrm{Lin}^{1}$ \\ ${ }^{1}$ Department of Physics, The Hong Kong University of Science and Technology, Kowloon 999077, Hong Kong, China \\ ${ }^{2}$ Division of Physical Sciences, South University of Science and Technology of China, Shenzhen 518055, China \\ *Corresponding author(email: jingliu@ust.hk)
}

\begin{abstract}
The self-assembly of indeno[1,2-b]fluorene-6,12-dione (IFDO) absorbed on Au(111) was investigated by scanning tunneling microscopy, scanning tunneling spectroscopy, and density functional theory calculations. It was found that IFDO molecules assembled into one-dimensional molecular chains along the herringbone structures on the $\mathrm{Au}(111)$ surface. The lowest unoccupied molecular orbital of IFDO molecules in the assembled structures, relative to isolated molecules, shifts towards Fermi level. The degree of molecular orbital shift, varying from 0.16 to $0.32 \mathrm{eV}$, depends on the pattern and number of hydrogen bonds formed between the detected IFDO molecule and its neighboring ones. Both transient and induced polarization of neighboring IFDO molecules contribute to the total polarization energy which leads to molecular orbital shift observed by experiments. The former makes the dominant contribution, while the effect of the latter is appreciable especially for molecules composing the defect structures.
\end{abstract}

Keywords: hydrogen bonds, molecular orbital shift, scanning tunneling microscopy, scanning tunneling spectroscopy, molecular polarization

doi: $10.1360 / \mathrm{N} 032018-00147$ 